\title{
Design of a Polymer-Based Hollow-Core Bandgap Fiber for Low-Loss Terahertz
} Transmission

Barh, Ajanta; Varshney, Ravi K.; Pal, Bishnu P.; Agrawal, G. P.; Rahman, B. M. A.

Published in:

I E E E Photonics Technology Letters

Link to article, DOI:

10.1109/LPT.2016.2544198

Publication date:

2016

Document Version

Peer reviewed version

Link back to DTU Orbit

Citation (APA):

Barh, A., Varshney, R. K., Pal, B. P., Agrawal, G. P., \& Rahman, B. M. A. (2016). Design of a Polymer-Based Hollow-Core Bandgap Fiber for Low-Loss Terahertz Transmission. I E E E Photonics Technology Letters, 28(15), 1703-1706. https://doi.org/10.1109/LPT.2016.2544198

\section{General rights}

Copyright and moral rights for the publications made accessible in the public portal are retained by the authors and/or other copyright owners and it is a condition of accessing publications that users recognise and abide by the legal requirements associated with these rights.

- Users may download and print one copy of any publication from the public portal for the purpose of private study or research.

- You may not further distribute the material or use it for any profit-making activity or commercial gain

- You may freely distribute the URL identifying the publication in the public portal 


\section{City Research Online}

\section{City, University of London Institutional Repository}

Citation: Barh, A., Varshney, R. K., Pal, B. P., Agrawal, G. P. \& Rahman, B. M. (2016). Design of a Polymer-Based Hollow-Core Bandgap Fiber for Low-Loss Terahertz Transmission. IEEE Photonics Technology Letters, 28(15), pp. 1703-1706. doi: 10.1109/LPT.2016.2544198

This is the accepted version of the paper.

This version of the publication may differ from the final published version.

Permanent repository link: http://openaccess.city.ac.uk/16551/

Link to published version: http://dx.doi.org/10.1109/LPT.2016.2544198

Copyright and reuse: City Research Online aims to make research outputs of City, University of London available to a wider audience. Copyright and Moral Rights remain with the author(s) and/or copyright holders. URLs from City Research Online may be freely distributed and linked to.

City Research Online: $\quad \underline{\text { http://openaccess.city.ac.uk/ publications@city.ac.uk }}$




\title{
Design of a polymer-based hollow-core band- gap fiber for low-loss terahertz transmission
}

\author{
Ajanta Barh, Ravi K. Varshney, Bishnu P. Pal, Senior Member, IEEE, G. P. Agrawal, Fellow, IEEE, \\ and B. M. A. Rahman, Fellow, IEEE
}

\begin{abstract}
We use numerical simulations to design a hollow-core microstructured polymer optical fiber (HC-mPOF) suitable for broadband, terahertz (THz) pulse transmission with relatively low losses and small dispersion. The HC-mPOF consists of a central large aircore surrounded by periodically arranged wavelength-scale circular air holes in a hexagonal pattern, embedded in a uniform Teflon matrix. The THz guidance in this fiber is achieved by exploiting the photonic band-gap (PBG) effect. In our low index contrast Teflon-air (1.44:1) hexagonal periodic lattice, the PBG appears only for a certain range of non-zero values of the longitudinal wave-vector. We have achieved PBG over a broad spectral range (band-width $\sim 400$ GHz) ranging from 1.65 to $2.05 \mathrm{THz}$ in the proposed HC-mPOF. The achievable loss coefficient in our designed HC-mPOF is $<4 \mathrm{~m}^{-1}$ and the group velocity dispersion parameter is $< \pm 5 \mathrm{ps} / \mathrm{THz} . \mathrm{cm}$ over a 300-GHz bandwidth (1.65 $1.95 \mathrm{THz})$.
\end{abstract}

Index Terms - Microstructured fiber, photonic band-gap, polymer fiber, terahertz wave.

\section{INTRODUCTION}

$I_{a}$ $\mathrm{N}$ recent years, terahertz $(\mathrm{THz})$ frequencies $(0.1$ to $10 \mathrm{THz})$ [1], a range that falls in between the far-infrared and microwaves, have assumed considerable importance due to their potential applications in medical diagnostics, tomography, homeland security, identification of hidden objects, defense, sensing, astronomy etc. [1-7]. Moreover, THz waves are being considered for data transmission including wireless transmission for integration into the existing communication bands. Unfortunately, this apparently promising radiation suffers from high propagation loss in the atmosphere (mainly due to absorption by water), which tends to vary with daily weather, climate, and altitude [8]. However, in the case of local-area networks, THz-wireless communication has shown some promising results $[9,10]$.

A solution for integration of the $\mathrm{THz}$ technology into the

Manuscript received ............ This work was supported in part by Trilateral UKIERI project entitled "Design and analysis of optical microstructured fiber based THz waves for transmission and applications."

A. Barh is now with Department of Photonics Engineering, DTU Fotonik, Denmark (email: ajaba@fotonik.dtu.dk)

R. K. Varshney is with the Department of Physics, IIT Delhi, Hauz Khas, New Delhi 110016, India (e-mail: ravi@physics.iitd.ac.in).

B. P. Pal is now with the School of Natural Sciences, Mahindra École Centrale College of Engineering, Hyderabad 500043, India (e-mail: bishnupal@gmail.com).

G. P. Agrawal is with the Institute of Optics, University of Rochester, Rochester, NY 14627, USA (e-mail: govind.agrawal@rochester.edu).

B. M. Azizur Rahman is with the School of Engineering and Mathematical Sciences, City University London, London EC1V 0HB, UK (e-mail: b.m.a.rahman@city.ac.uk).

Color versions of one or more of the figures in this paper are available online. communication band is to develop $\mathrm{THz}$ waveguides. However, this frequency range is not compatible with most conventional materials. Unlike optical waves, $\mathrm{THz}$ radiation suffers high absorption losses in conventional dielectric waveguides, whereas at the same time, unlike microwaves, $\mathrm{THz}$ radiation also suffers from high Ohmic losses and dispersion in metallic WGs [11]. Interestingly, though a $\mathrm{THz}$ wave suffers high losses in metal, glass, water, dust, fog, and cloud, it can penetrate deep into dry air, plastics, cloths, ceramics etc., which could potentially be utilized as a guiding medium for the $\mathrm{THz}$ radiation with appropriate engineering. Moreover, suitable THz WGs will also prove valuable for applications such as, remote sensing, medical endoscopy, diffraction-limited guidance, collimated beam delivery, long distance broad-band communication, and stronger light-matter interaction.

In the recent past several proposals for $\mathrm{THz}$ wave guidance have been explored using metallic or metal-dielectric based hybrid WG structures. Early THz WGs were composed of planar structures, where coplanar transmission lines, common for microwaves, were used to guide $\mathrm{THz}$ waves [12]. However, they suffer from high propagation loss $\left(\sim 20 \mathrm{~cm}^{-1}\right)$ at $\mathrm{THz}$ frequencies from the combined effects of Ohmic, diffraction and radiation losses. More recently, non-planar structures with both metals as well as few selective dielectric materials had been explored to design the $\mathrm{THz}$ WGs. The metallic WGs are the scaled-down version of microwave WGs, whereas the dielectric WGs are scaled-up version of the optical ones. Metallic non-planar WGs such as hollow core, parallel plate, metal sheet, single wire, two-wire structure, slit-WG etc. have also been reported [11, 13, 14]. Depending on the nature of the modes (TE/TM/TEM) and their confinement, the overall loss varies, and the dispersion increases drastically near the cut-off frequencies. In the case of dielectricWGs also a trade-off exists between confinement and material loss. However, unlike the metallic WGs, dielectric WGs can take the form of an optical fiber, and the primary challenge is to find suitable materials for such a $\mathrm{THz}$ fiber. Dielectric polymers such as high-density polyethylene (HDPE), cyclic olefin copolymer (COC), polyethylene (PE), polytetrafluoroethylene (Teflon) are highly transparent and possess flat material dispersion over a wide $\mathrm{THz}$ band $[11,15]$. Moreover, these polymers can be drawn in a fiber form [11, 16]. Different kinds of index-guided polymer fibers have already been proposed in the literature for $\mathrm{THz}$ guidance. Though they support broad-band transmission, their primary limiting factor is the material loss [11]. Dry air is perhaps the lowest transmission loss material for $\mathrm{THz}$ waves. From this perspective, a hollow-core fiber structure should be an effective solution provided that either a photonic band-gap (PBG) or an anti-resonance reflective (ARR) guiding mechanism can be used 
for confining the mode within the air-core. Recently several types of hollow-core THz fibers (Bragg fiber, microstructured fiber and ARR-guided structure) have been proposed with low dispersion and low loss [11, 17-19]. The primary drawback of these schemes is that their transmission window is limited by the PBG effect and to achieve low loss, the overall cross-section often exceeds tens of $\mathrm{mm}$, which makes them bulky and inflexible.

In this paper, we report a numerically designed hollow-core microstructured polymer optical fiber (HC-mPOF) for low-loss guided transmission of $\mathrm{THz}$ radiation. The transmission loss is minimized by maximizing the confinement of the modal field within the air-core and reducing the overall fiber cross-section to improve its flexibility. The fiber cladding is made of Teflon with air holes in a hexagonal lattice structure. We have first examined the criteria for the existence of a PBG in such a structure and found after optimization that a PBG covering a wide band-width (> $400 \mathrm{GHz}$, ranging from $1.65 \sim 2.05 \mathrm{THz}$ ) can be realized for the fundamental guided mode. We discuss the overall loss and dispersion characteristics of the designed structure in detail. Our designed parameters should be useful for motivating fabrication of $\mathrm{THz}$ transmission fiber.

\section{NUMERICAL MODELING}

In general, a PBG fiber is formed by introducing wavelengthscale periodic refractive index (RI) features over its transverse cross-section (say $x-y$ plane) all along the fiber length ( $z$ axis). If we create a defect region in that otherwise periodic medium, the PBG-guided mode will be confined to that region (mimicking as a fiber core). In a two-dimensional (2-D) periodic lattice, the solution of Maxwell's equation can be written as [20]

$$
\mathbf{H}_{\left(\mathrm{n}, \mathrm{k}_{\mathrm{z}}, \mathrm{k}_{0}\right)}(\mathbf{r})=\mathbf{u}_{\left(\mathrm{n}, \mathrm{k}_{\mathrm{z}}, \mathrm{k}_{0}\right)}(\boldsymbol{\rho}) e^{i \mathbf{k}_{0} \cdot \boldsymbol{\rho}} e^{i \mathrm{k}_{\mathrm{z}} \mathrm{z}}
$$

where, $\mathbf{H}$ is the magnetic field, $\mathbf{r}$ is the position vector, $\boldsymbol{\rho}$ is the projection of $\mathbf{r}$ on the $x-y$ plane, $\mathbf{k}_{0}$ is the in-plane ( $x-y$ plane) wave-vector, lying inside the Brillouin Zone, $\mathrm{k}_{\mathrm{z}}$ is the longitudinal ( $z$ ) wave-vector, " $n$ " is the band number and $\mathbf{u}(\boldsymbol{\rho})$ is a periodic function satisfying the relation, $\mathbf{u}(\boldsymbol{\rho})=\mathbf{u}(\boldsymbol{\rho}+\mathbf{R})$, for any lattice vector, $\mathbf{R}$ in the $x-y$ plane.

Though the PBG effect in 1-D periodic structures (e.g., Bragg fibers) can be studied analytically [21], a 2-D structure requires more complex analysis, and for accurate results, numerical modeling is inevitable. Full-vector plane wave expansion (PWE) is a popular numerical technique for calculating the band-structure of any complex geometry. However, it cannot handle losses and frequency dependent dispersion of the structure. In contrast, the finite element method (FEM) is more complicated numerically but it can handle both the loss and dispersion quite accurately. We employ both of these techniques. First, we employed the PWE method for locating the PBG in the periodic lattice, ignoring the loss and dispersion. After that, we defined a super lattice structure, which includes both the defect core and surrounding microstructured cladding, and then, investigated the loss and dispersion characteristics of the PBG-guided mode via FEM.

\section{PROPOSED FIBER DESIGN}

The base material for the proposed fiber structure is chosen to be Teflon, as it is chemically inert to most chemicals, suitable for fiber drawing, transparent over a wide $\mathrm{THz}$ band and a relatively low cost material. The fiber's cross-section is composed of a uniform background of high index Teflon (RI about 1.44) with hexagonally arranged 4 rings of circular air holes $(\mathrm{RI}=1)$ embedded in it. The diameter of air holes and their centre to centre separation are denoted as $d$ and $\Lambda$, respectively. The central 7 airholes are replaced by a bigger hole to create the defect hollowcore, which is then surrounded by a 3 periodic cladding rings. The cross-section of the proposed HC-mPOF is shown in Fig. 1. The RI of Teflon provides lower index contrast with air in comparison to other THz compatible polymers. Thus, the air-Teflon structure provides lower scattering loss at the dielectric boundaries (according to Ref. [22]). However, this low index contrast structure $\left[\Delta \approx\left(n_{1}-n_{2}\right) / n_{1} \approx 0.31\right]$ cannot open a PBG for the inplane wave-vector of either the TE or TM mode, at least in such a simple hexagonal geometry [cf. Fig. 2(a)]. Interestingly, a nonzero value of the longitudinal propagation constant (normalized value is $\beta_{\mathrm{p}}=k_{\mathrm{z}} n_{\mathrm{eff}} \Lambda$, where $n_{\mathrm{eff}}$ is the effective index of the fiber mode) smoothens the band edges of in-plane $(x-y)$ band-structure. As a result, a suitable range of $\beta_{\mathrm{p}}$ opens up one or more PBGs for such a 2-D structure [cf. Fig. 2(b)].

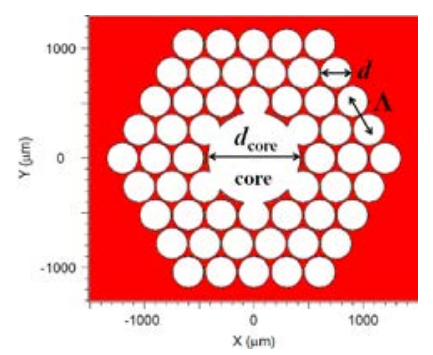

Fig. 1. Transverse cross section of the proposed HC-mPOF. Cladding is formed by the three hexagonally arranged rings of circular (shown in white) air holes (of diameter $d$ and pitch $\Lambda$ ) embedded in Teflon (red background). The core at the center is formed by a circular air hole of bigger diameter, $d_{\text {core }}$.
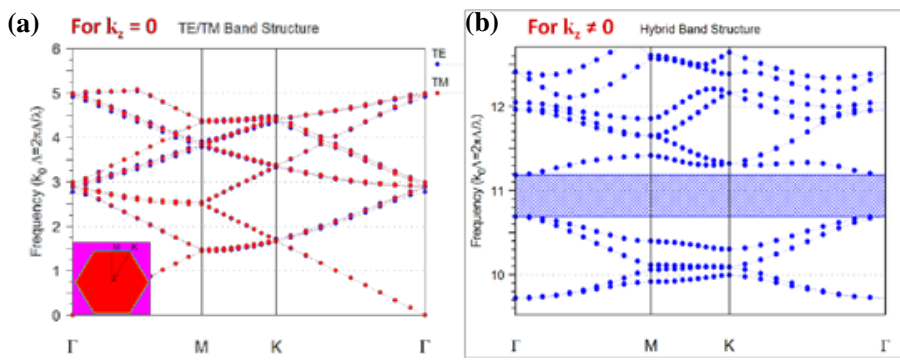

Fig. 2. Band diagram of air-Teflon hexagonal lattice for (a) $k_{z}=0$, and (b) $k_{z} \neq 0$. The blue shaded region corresponds to lowest order band-gap. The inset in (a) shows the $1^{\text {st }}$ Brillouin zone indicating co-ordinates of the k-vector.

The cross-section of the HC-mPOF is optimized by investigating its band-structure by the PWE method using the commercially available software $\mathrm{RSoft}^{\circledR}$. Although higher values of $d / \Lambda$ can yield a stronger band-gap effect, we cannot increase it arbitrarily as very high values of $d / \Lambda$ pose difficulties in fabrication. By taking into consideration of these issues we have fixed it at 0.94, as several microstructured polymer fibers with such a large air-fraction has already been reported in the literature [23]. Thereafter we investigated scalability of this band-gap by varying the pitch $(\Lambda)$. For smaller values of $\Lambda$, the bang-gap effect becomes stronger so the gap-width increases; however, the midgap wavelength reduces due to a smaller periodicity. For further study, we have fixed the value of $\Lambda$ at $300 \mu \mathrm{m}$, which provides us with a band-gap centered near $1.8 \mathrm{THz}$ and with a band-width of more than $100 \mathrm{GHz}$. 


\section{RESUlTS AND DisCUSSION}

The band-structure of the proposed periodic cladding structure is shown in Fig. 2(b) for a fixed value of $\beta_{\mathrm{p}}$. However, we have also varied the z-component of the wave-vector to produce a complete map of band-gap (mainly for the lowest order band-gap) as a function of $\beta_{\mathrm{p}}$, as seen in Fig. 3. The green shaded regions are the gap map, which are gradually increasing in width for higher values of $\beta_{\mathrm{p}}$ as it flattens the band-edges more. The light line corresponding to the core RI is also superposed on the figure (solid line in red). For our proposed air-core structure the light line is essentially a straight line with unit slope in the frequency vs wave-vector plane. The bound modes of interest, which are basically the defect states of the transverse periodic lattice, must confine inside the hollow-core, and hence its dispersion relation must follow (more or less!) this light line. The encircled region with dashed line (c.f. Fig. 3 ) indicates the range of $\beta_{\mathrm{p}}(10.2 \sim 11.5)$ and frequency for which the bound modes can exist. This range can be understood physically as follows: smaller values of $\beta_{\mathrm{p}}$ are not sufficient to open up the PBG for bound modes, whereas, for larger values of $\beta_{\mathrm{p}}$, the fields of modes become more confined in the high RI region, which reduces overlaps among them and as a consequence the PBG disappears.

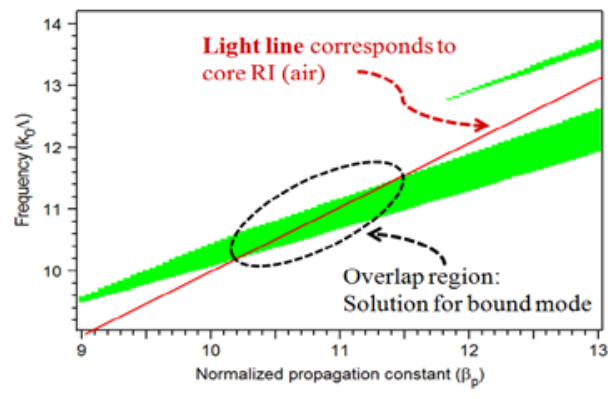

Fig. 3. Hybrid gap map (green shaded regions) for different $\beta_{\text {p's. Solid line in red }}$ represents the light line corresponding to the core RI. Overlap region (indicated by the oval shape in black dash line) represents the solution for bound modes.

At the next step we study the bound modes (PBG guided modes) by considering a supercell geometry. The supercell, consists of multiple unit cells including the defect core at the center, now becomes the new unit cell. However, the size of this new cell must be large enough to sufficiently isolate the defect core for accurate results. The core size is chosen carefully to eliminate the unwanted surface states [20], which are generally a big hindrance to achieve low transmission loss in PBG fibers. By comparing the performance between different core diameters $\left(d_{\text {core }}\right)$, we chose $d_{\text {core }}=2.8 \Lambda$, which resulted in a non-uniform core-surface boundary. As a consequence, the surface states get eliminated significantly and the confinement loss is minimized.

To calculate the loss and dispersion of the fundamental bound mode, we employed the FEM in a commercially available FEMSIM module in RSoft ${ }^{\circledR}$ software. In the FEM analysis, we have employed an appropriate perfectly matched layer (PML) and optimized its width along with the FEM element size to yield a converging solution. A fine triangular element of size $=\Lambda / 64$ is considered throughout the FEM analysis. In this context, we have scanned the imaginary part of the mode's effective index [ $\left.\operatorname{Img}\left(n_{\text {eff }}\right)\right]$ as a function of PML width [see Fig. 4(a)], which reveals that, converging solution can be obtained for PML widths $>10 \mu \mathrm{m}$. As a precautionary measure, we have fixed its value at $30 \mu \mathrm{m}$ for rest of the analysis. The mode profile of the fundamental PBG guided mode at $1.7 \mathrm{THz}$ is plotted in Fig. 4(b), where only the $1^{\text {st }}$ quadrant of simulation domain is shown owing to the structural symmetry of our fiber. The figure clearly reveals a strongly confined fundamental mode (nearly Gaussian in shape) in the proposed HC-mPOF. We may mention that a higher order mode also coexists with the fundamental one. However, its confinement loss is larger by a factor of 1000 , indicating that it would leak away after a short distance of propagation.
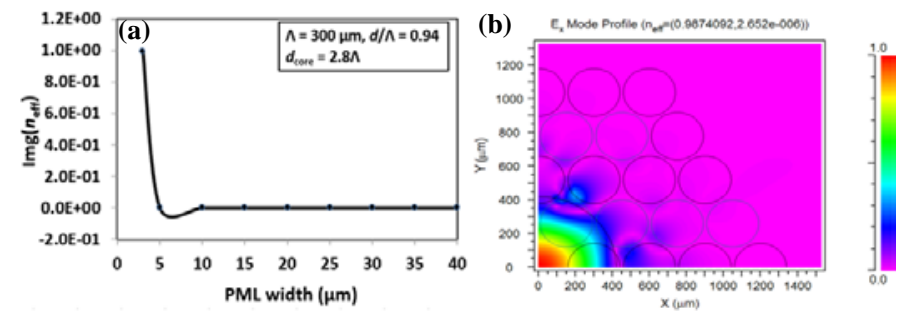

Fig. 4. (a) Variation of the imaginary part of the effective index as a function of PML width. (b) 2-D mode field profile ( $x$ component of the $E$-field) of the fundamental mode; only $1^{\text {st }}$ quadrant is plotted owing to an obvious symmetry in the structure.

Total transmission loss is a combined effect of three major sources of loss: confinement loss $\left(\alpha_{c}\right)$, material absorption loss $\left(\alpha_{\mathrm{m}}\right)$ and scattering loss $\left(\alpha_{\mathrm{s}}\right)$. Since the RI contrast is relatively small for our structure, $\alpha_{\mathrm{s}}$ would be relatively low, and hence we have neglected it in the calculation of transmission loss. The $\alpha_{\mathrm{c}}$ arises from the finite number of air hole rings in the cladding and can be calculated from the $\operatorname{Img}\left(n_{\text {eff }}\right)$ component of the corresponding mode. We have calculated the transmission loss with and without material absorption, and the results are shown in Fig. 5(a). A fixed value of material loss for Teflon $\left(0.8 \mathrm{~cm}^{-1}\right)$ was used in our calculations [24]. The figure reveals that the material loss of Teflon has relatively small effect on the overall loss spectrum. This is because the mode is strongly confined within the hollow core and has very little overlap with the Teflon structure. As a result, a loss of less than $0.04 \mathrm{~cm}^{-1}$ can be achieved in our proposed fiber over a wide range of $1.65 \sim 2.05 \mathrm{THz}$ (bandwidth $400 \mathrm{GHz}$ ) even after including the material loss. We note that it can be reduced even further by increasing the number of air hole rings. An expanded view of loss spectrum is shown in the inset of Fig. 5(a). For optical pulses propagating through an optical fiber, group velocity dispersion (GVD) plays a crucial role as it leads to distortion of pulse shape and pulse broadening. It is thus important to consider the GVD of our designed fiber for $\mathrm{THz}$ frequencies. Fortunately, as the mode is strongly confined inside the hollowcore, it experiences very low dispersion within the band-gap; however, similar to any other PBG-guided structure, the dispersion increases sharply near the band-edges. We have calculated the GVD parameter $\left(\beta_{2}\right)$ from the frequency dependence of the real part of the mode effective index $\left[\operatorname{Re}\left(n_{\text {eff }}\right)\right]$, and the results are shown in Fig. 5(b). The GVD is anomalous $\left(\beta_{2}\right.$ $<0)$ except for a small frequency window $(1.8 \sim 1.87 \mathrm{THz})$, where it becomes normal $\left(\beta_{2}>0\right)$. However, the amplitude of $\beta_{2}$ remains below $5 \mathrm{ps} /(\mathrm{THz} . \mathrm{cm})$ over the band-gap regions, which is indeed quite low, and it blows up at both the band-edges. 

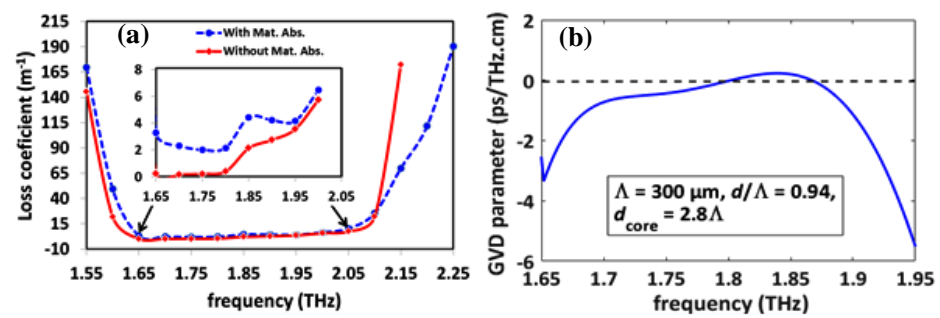

Fig.5. (a) Variation of transmission loss [with (blue dashed) and without (red solid) material absorption] and (b) GVD parameter as a function of THz frequency for the fundamental mode of the proposed HC-mPOF. The inset in 'a' shows an expanded view of the two loss spectra.

\section{CONCLUSION}

In this paper, design of a PBG fiber is proposed for possible application in $\mathrm{THz}$ wave transmission. Through a detailed numerical analysis, a HC-mPOF is optimized to yield a PBG around $1.8 \mathrm{THz}$. The primary aim was to maximize the $\mathrm{THz}$ transmission window while minimizing both the loss and dispersion, and reduce the overall fiber cross-section for a compact design. The cross-section of the proposed HC-mPOF consists of a hollow-core (defect region), surrounded by hexagonally arranged 3 rings of air holes embedded in a uniform higher index material (Teflon). The size of the fiber core is deliberately chosen larger from rest of the holes and its diameter is optimized to 2.8 times the lattice periodicity to achieve PBG for out of plane propagation and to eliminate the unwanted lossy surface-states with sufficient mode confinement. The structure is first optimized through the plane wave expansion method to locate the band-gap and study its scalability. After optimizing the crosssection, a finite element method is employed to thoroughly investigate the loss and dispersion characteristics of the PBG guided mode. Our results reveal that just 3 air-hole rings in the cladding are enough to establish single mode guidance with extremely low confinement loss. The calculated PBG extends over almost $400 \mathrm{GHz}$ (range $1.65 \sim 2.05 \mathrm{THz}$ ) band-width with very low transmission losses $\left(\leq 4 \mathrm{~m}^{-1}\right)$ and low GVD $\left(\beta_{2}< \pm 5\right.$ ps/THz.cm) over $1.65-1.95 \mathrm{THz}$. The nearly Gaussian shaped fundamental mode is strongly confined inside the hollow core. Though in general, the fabrication of hollow-core fibers is difficult, recently reported modern fabrication technologies [11, $16,18,19,23,25]$ show lot of promise to realize such complex fiber structures in practice. Our proposed HC-mPOF should be a good candidate for high-power, low-loss, low-dispersion pulse propagation in the $\mathrm{THz}$ regime and may prove valuable for a multitude of applications.

Preliminary results of this study were reported at the OSA endorsed conference Photonics 2014, held in December 2014 in Kharagpur, India.

\section{REFERENCES}

[1] X. C. Zhang, "Terahertz wave imaging: horizons and hurdles," Phys. Med. Biol., vol. 47, no. 21, pp. 3667-3677, Oct., 2002.

[2] C. Kulesa, "Terahertz spectroscopy for astronomy: From comets to cosmology,” IEEE Trans. on Terahertz Sc. and Technol., vol. 1, no. 1, pp. 232-240, Sept., 2011

[3] G. J. Wilmink and J. E. Grundt, "Invited review article: current state of research on biological effects of terahertz radiation," J. Infrared, Millimeter, and Terahertz Waves, vol. 32, no. 10, pp. 1074-1122, Jun, 2011.

[4] P. H. Siegel, "Terahertz technology in biology and medicine," in IEEE Microwave Symposium Digest, 2004, pp. 1575-1578.
[5] H. H. Mantsch and D. Naumann, "Terahertz spectroscopy: The renaissance of far infrared spectroscopy,” Journal of Molecular Structure, vol. 964, no. 1, pp. 1-4, Feb., 2010.

[6] M. Kowalski, M. Kastek, M. Piszczek, M. Życzkowski, and M. Szustakowski, "Harmless screening of humans for the detection of concealed objects," in Safety and Security Engineering VI, WIT press, 2015, pp. 215223.

[7] J. F. Federici, B. Schulkin, F. Huang, D. Gary, R. Barat, F. Oliveira, and D. Zimdars, "THz imaging and sensing for security applications-explosives, weapons and drugs," Semiconductor Sc. and Technol., vol. 20, no. 7, pp. S266-S280, Jul., 2005.

[8] Y. Yang, M. Mandehgar, and D. Grischkowsky, "Understanding THz pulse propagation in the atmosphere," IEEE Trans. on Terahertz Sc. and Technol., vol. 2, no. 4, pp. 406-415, Jul., 2012.

[9] T. Kleine-Ostmann and T. Nagatsuma, "A review on terahertz communications research," Journal of Infrared, Millimeter, and Terahertz Waves, vol. 32, no. 2, pp. 143-171, Jan., 2011.

[10] S. Koenig, D. Lopez-Diaz, J. Antes, F. Boes, R. Henneberger, A. Leuther, A. Tessmann, R. Schmogrow, D. Hillerkuss, R. Palmer, T. Zwick, C. Koos, W. Freude, O. Ambacher, J. Leuthold, and I. Kallfass, "Wireless sub-THz communication system with high data rate," Nat. Photonics, vol.7, no. 12, pp. 977-981, Oct., 2013.

[11] S. Atakaramians, V. S. Afshar, T. M. Monro, and D. Abbott, "Terahertz dielectric waveguides," Advances in Optics and Photonics, vol. 5, no. 2, pp. 169-215, Jul., 2013.

[12] M. Y. Frankel, S. Gupta, J. A. Valdmanis, and G. A. Mourou, "Terahertz attenuation and dispersion characteristics of coplanar transmission lines," IEEE Trans. on Microwave Theory and Techniques, vol. 39, no. 6, pp. 910916, Jun., 1991.

[13] G. Gallot, S. P. Jamison, R. W. McGowan, and D. Grischkowsky, "Terahertz waveguides," JOSA B, vol. 17, no. 5, pp. 851-863, May, 2000.

[14] H. Pahlevaninezhad, T. E. Darcie, and B. Heshmat, "Two-wire waveguide for terahertz,” Opt. Exp., vol. 18, no. 7, pp. 7415-7420, Mar., 2010.

[15] http://www.tydexoptics.com/products/thz_optics/thz_materials/

[16] M. van Eijkelenborg, M. Large, A. Argyros, J. Zagari, S. Manos, N. Issa, I. Bassett, S. Fleming, R. C. McPhedran, C. M. de Sterke, and N. A. Nicorovici, "Microstructured polymer optical fibre," Opt. Exp., vol. 9, no. 7, pp. 319-327, Sept., 2001

[17] C. S. Ponseca Jr, R. Pobre, E. Estacio, N. Sarukura, A. Argyros, M. C. Large, and M. A. van Eijkelenborg, "Transmission of terahertz radiation using a microstructured polymer optical fiber,” Opt. Lett., vol. 33, no. 9, pp. 902904, May, 2008.

[18] L. Vincetti, "Hollow core photonic band gap fiber for THz applications," Microwave and Opt. Technol. Lett., vol. 51, no. 7, pp. 1711-1714, Jul., 2009.

[19] J. Anthony, R. Leonhardt, S. G. Leon-Saval, and A. Argyros, "THz propagation in kagome hollow-core microstructured fibers," Opt. Exp., vol. 19, no. 19, pp. 18470-18478, Sept., 2011.

[20] J. D. Joannopoulos, S. G. Johnson, J. N. Winn, and R. D. Meade, Photonic crystals: molding the flow of light, Princeton University press, Princeton and Oxford, (2011).

[21] S. Dasgupta, B. P. Pal, and M. R. Shenoy, "Photonic bandgap-guided Bragg fibers," in Guided Wave Optical Components and Devices, Academic press, 2005, pp. 71-82.

[22] T. Barwicz and H. A. Haus, "Three-dimensional analysis of scattering losses due to sidewall roughness in microphotonic waveguides," J. Lightwave Technol., vol. 23, no. 9, pp. 2719-2732, Sept., 2005.

[23] A. Argyros, "Microstructured polymer optical fibers," J. Lightwave Technology, vol. 27, no. 11, pp. 1571-1579, Jun., 2009.

[24] C. Winnewisser, F. Lewen, and H. Helm, "Transmission characteristics of dichroic filters measured by THz time-domain spectroscopy,” Appl. Phys. A: Materials Sc. \& Processing, vol. 66, no. 6, pp. 593-598, Jun., 1998.

[25] P. Laurin, M. Girard, A. Markov, M. Skorobogatiy, "Hollow core terahertz optical fibers with hyperuniform disordered dielectric reflectors," in Proc. IEEE IRMMW-THz, 2014, pp. 1-2. 